\title{
Progress in Environment Friendly Nano- Technologies
}

\author{
Oleg L Figovsky* and Blank N \\ Israel Association of Inventors, Israel \\ *Corresponding author: Oleg L Figovsky, Israel Association of Inventors, Israel,
}

Submission: 㘹 May 07, 2018; Published: 笽 October 05, 2018

\begin{abstract}
One of the most interesting and perspective directions in material engineering of the last years is development of technology of nanocomposite materials consisting from two or more phases with precise interphase border and nanostructured materials based on interpenetrated polymer network. Israel is one of leaders in nanotechnology, not only in fundamental academic researches but mainly in industrial researches and founding start-up companies Some important results in the nanotechnology material engineering field in Israel are summarized in the paper.
\end{abstract}

Keywords: Nanotechnology; Nanocomposites; Nanostructured material

\section{Introduction}

The economic, security, military and environmental implications of molecular manufacturing are extreme. Unfortunately, conflicting definitions of nanotechnology and blurry distinctions between significantly different fields have complicated the effort to understand those differences and to develop sensible, effective policy for each.

The risks of today's nanoscale technologies cannot be treated the same as the risks of longer-term molecular manufacturing. It is a mistake to put them together in one basket for policy consideration-each is important to address, but they offer different problems and will require far different solutions. As used today, the term nanotechnology usually refers to a broad collection of mostly disconnected fields. Essentially, anything sufficiently small and interesting can be called nanotechnology. Much of it is harmless. For the rest, much of the harm is of familiar and limited quality. Molecular manufacturing, by contrast, will bring unfamiliar risks and new classes of problems.

Desktop nanofactories will use vast arrays of tiny machines to fasten single molecules together quickly and precisely, allowing engineers, designers, and potentially anyone else to make powerful products at the touch of a button. Although such a contraption has been envisioned in some detail for almost two decades, and although the basic concept goes back to 1959, when the physicist Feynman first articulated it, it's only in recent years that technology has advanced to the point where we can begin to see the practical steps that might bring it into reality.
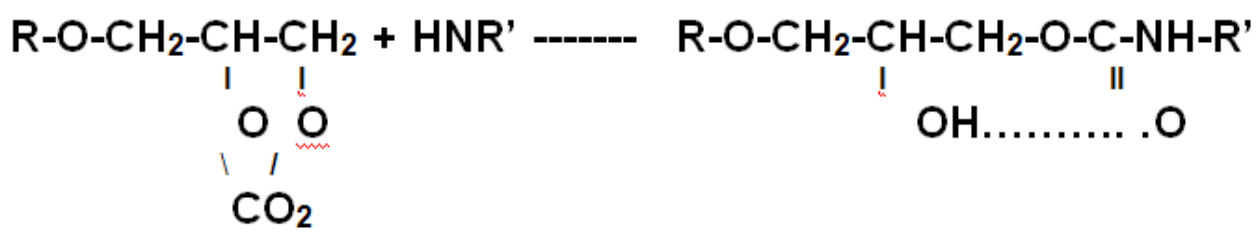

Figure 1

Figure 2

The essence of nanotechnology is the ability to work at the molecular level to create large structures with fundamentally new molecular organization. Materials with features on the scale of nanometers often have properties different from their macroscale counterparts. The prospect of a new materials technology that can function as a low-cost alternative to high-performance composites has, thus, become irresistible around the world. By this means nanotechnology presents a new approach to material science and 
engineering as well as for design of new devices and processes. Figure $1 \& 2$ can give some imagine of the global tendency of nanotechnology development [1-4].

As is known, composite materials are two- or multiphase with well-defined interphase border. Such materials contain the reinforcing elements immersed into a polymeric, ceramic or metal matrix. Mechanical properties of composites depend on structure and properties of the interphase border. Phases of usual composite materials have micron and submicron sizes.

Importantamong thesenanoscalematerialsarenanocomposites, in which the constituents are mixerd on a nanometer-length scale. They often have properties that are superior to conventional microscale composites and can be synthesized using surprisingly simple and inexpensive techniques. The tendency to reduction of the phase's sizes of a filler (a strengthening element) is attributable to decrease in its microscopic deficiency (the size of one of nanocomposite phases does not exceed 100nm) Due to the nanometer size of the particles, which is smaller than the wavelength of visible light, the reinforced polymer remains transparent. Other characteristics of the composites include high barrier performance and improved thermal stability, which make these compounds suitable for many applications. Because of this technology of nanocomposites is one of the most perspective directions in a material engineering. Specifically, nanocomposite technology is applicable to a wide range of polymers. Cutting across the material classes of thermoplastic, thermosets and elastomers.

\section{General Comment}

Our current developments currently underway include the following:

1. Nanostructured composites based on Interpenetrated Polymer Network;

2. Epoxy-rubber composites with nanoheterogenic structure;

3. Nanocomposites based on hybrid organo-silicate matrix;

4. Polymer nanocomposites with very low permeability and high resistance to aggressive environments;

5. Metal matrix nanocomposites produced by SDP method.

6. Polymer matrix nanocomposites and nanomembranes produced by SDP method;

7. Biodegradable materials based on Nanocellulose.

\section{Nanostructured Composites Based on Interpenetrated Polymer Network}

This project is oriented to prepare nanocomposites based on interpenetrated polymer network (IPN), such as polyurethanes, epoxies and acrylate by way of creating nanoparticles of $\mathrm{SiO}_{2}$, $\mathrm{TiO}_{2}$ and other metal oxides during a technological stage from a liquid phase. Using as interpenetrating polymer networks principle in production of composite materials provides a unique possibility to regulate their both micro-and nano-structured properties Formulation of a new class of nanocomposite materials is characterized by the absence of contaminants for a network polymers technology. As a main component of such technology we are using branched (dendro)-amino silanes that at the first stage are curing agents for many oligomers).

The proposed dendro-amino silane hardeners give the possibility to introduce the siloxane fragments into aromatic structure of diphenylolpropane based epoxy-amine network polymers. Additional hydrolysis of amino silane oligomer creates the secondary nano-structured network polymer that improves the service properties of the compound. Branched (dendro) polyamine hardeners are novel direction in epoxy and cyclocarbonate and acryl resins chemistry.

The new hardeners give rise to formation of IPN of a polymerized resin with a polysiloxane network by the hydrolytic polycondensation of silane groups. IPN network may be formed on the base of epoxy- cyclocarbonate oligomers. It was found that at least 0.1 equivalent weight of silane per epoxy resin equivalent weight may result in IPN formation. It has been known that epoxy resin has low resistance to acetone and methanol attack. IPN film provides increasing the resistance (Figure $3 \& 4$ ).

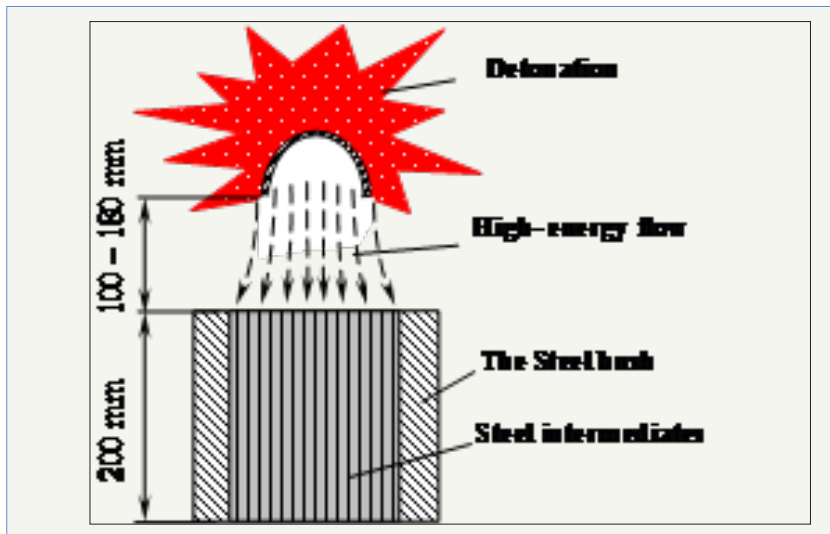

Figure 3: Principal scheme of superdeep penetration of micro particles into metal body.

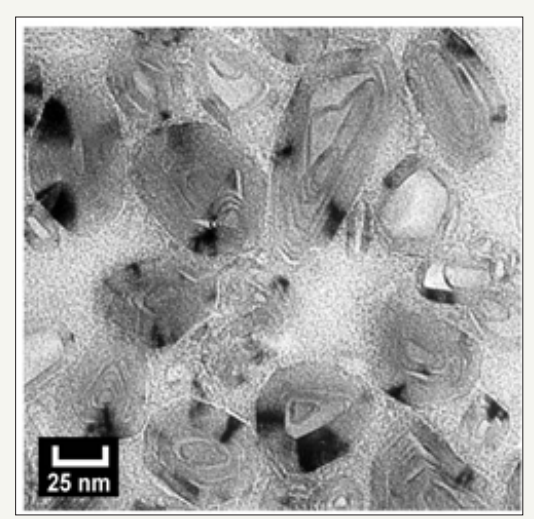

Figure 4: Transmission electron microscopy (TEM) photography of the astralenes in water suspension.

Novel hybrid nonisocyanate polyurethane based nanocomposites (HNIPU) was received by the following reaction. 
Pilot production of two component paints, top coatings, adhesives and floorings are obtained. Figure 5 illustrated industrial application of the IPN flooring. The two-component compound have unique properties that combine the best mechanical properties of polyurethane and chemical resistance of epoxy binders.

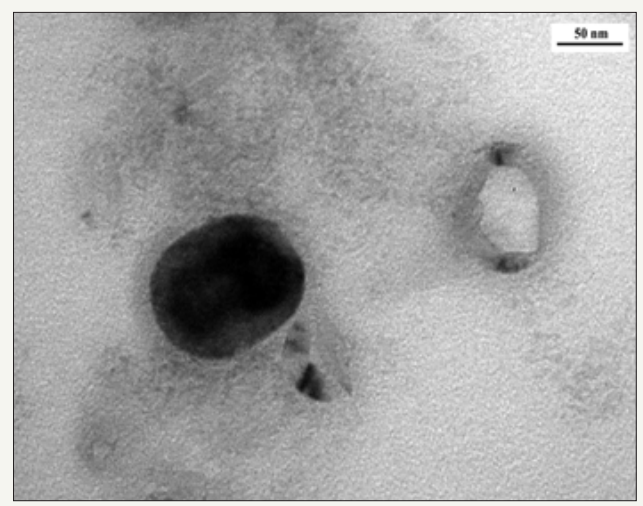

Figure 5: TEM-photography of initial phase of cement hydration with astralenes.

\section{Nanocomposites Based on Hybrid Organo-Silicate Matrix}

Important among nanoscale materials are hybrids or nanocomposites. They often exhibit properties superior to conventional composites. Organic-inorganic hybrid nanostuctures have generated great interest by combining optical, magnetic or electronic properties of inorganic crystals with mechanical properties and functionality of organic compounds. They suggest a variety of potential applications as electrical, optical and medicals markers.

By using a principle of forming nanostructure by creating nanoparticles during a technological process from a liquid phase, we has elaborated a few of composites based on different kinds of soluble silicates. Significant increasing of silicate matrix strength and toughness was reached by incorporation of special liquid additives, such as TFS, which effect as a micro-crystallizing nucleator on the technological stage and later they colmatage the pores of silicate matrix. Our last elaborations are mainly applying a novel type of soluble silicate contained organic cations, for example, the DABCO

based organic alkali soluble silicate.

\section{Polymer nanocompsites with very low permeability and high resistance to aggressive environments}

Novel chemically resistant polymer materials were elaborated with adding nano-size inorganic active fillers that react with aggressive medium into which they are introduced, forming a new phase of high-strength hydrate complexes. This enhanced bonding occurs upon the penetration of aggressive media into active nano-fillers containing polymer material. The chemical resistant properties of the forming polymer materials are activated by harsh environmental conditions where polymer systems without additives remain defenseless to chemical corrosion. We have developed an extensive product range of such active nano-fillers for upgrading the most common polymers against a wide variety of aggressive media including acids, sea water, fluorine, alkalis and more.

\section{Novel metalic \& polimer matrix nanoreinforced materials produced by method of superdeep penetration}

Technological process on the basis of new physical effect "superdeep penetration" (SDP) allows to make from the known tool steels (for example, HSS) new composite materials (Figure 3). These materials can be used for replacement base steels in metalcutting and stamp tools. In some cases, new materials can be used for replacement of a hard metal (on the basis of WC) in the tool for mining (for example, cutters of coal and mining machines). The application of the new SDP technology allows to increase the service life of the tools up to 1.5-5.0 times compared to the commonly used tools. The technology can be applied for the volume strengthening practically any type of instrumental steels.

Use of new physical effect SDP allows as well to obtain the polymeric nano-menbranes by using as a penetrated agent water solution of soluble salts.

\section{Water-dispersion paint composition with biocide properties based on silver nano-powder}

We have elaborated advanced bioactive coating with using silver nanoparticles. As found in numerous studies during the last two decades, particles with dimensions in nanometer scale $\left(10^{-9}\right.$ $10^{-8} \mathrm{~m}$ ) possess peculiar properties, different from those of atoms and ions on the one hand and of bulk substance on the other. These silver nanoparticles were received by the novel BAR-synthesis. The biological activity of varnish-paint materials modified by silver nanoparticles was estimated on the following microorganisms:

1. Escherichia coli (E. Coli 1257) as a conventional device. The effect from use of a new aluminum material in electric installations and electronic control systems will make, due to reduction in expenses for expensive materials, hundred million and billions dollars USA. Cost of process of rearrangement of structure of aluminium preparation does not exceed 40USD $/ \mathrm{kg}$. By industrial production of such material its cost to decrease in 2-3 times. From individual preparation can be made tens electric and thousand electronic devices. Process SDP is high-efficiency and does not demand the expensive equipment. The new technology of volumetric reorganization of aluminum, creation zones of nanostructures, the materials received on this basis, will find wide application by manufacture of electric installations and electronic devices. model of bacterial contamination of the environment;

2. Coliphage (RNA-phage MS-2) as a model of viral infection, including influenza A and B, hepatitis A

3. Mold fungi (Penicillinum Chrysogenum) as a typical representative of microflora of the dwellings and a model of fungicidal contamination;

4. Spores as a model of spores and other microflora. 
The data of the testing confirms the significant advantages of elaborated water-born acrylic bioactive coatings.

\section{Nanocellulose and Biodegrable Composite Materials}

(NanoCell) with CI crystalline modification was prepared using advanced, environmentally friendly, resource-save and cheap technology. The developed technology permits producing NanoCell in pilot and industrial amounts. NanoCell product can be manufactured in the form of dispersions, high solid paste and dry powder.

The FDA-approved aqueous polymer nanostructured composition CreenCoat is applied for protective covering of paper and board. The coating layer imparts to material barrier properties against permeation of water, grease, oxygen and some other substances. The GreenCoat emulsion is coated on cardboard surface by means of bar - coater and dried at temperature $150-170{ }^{\circ} \mathrm{C}$ for $30-60$ sec. Then the GreenCoat $\mathrm{W}$ glazing hot melt composition is coated on first layer by means of bar-coater at temperature 130 $135{ }^{\circ} \mathrm{C}$ and air cooled. Waste of coated material can be repulped and used in paper industry or decomposed in nature due to its biodegradability.

\section{Composite materials modified by astralenes}

Power characteristics of electric field on a surface of nonmetallic particles in applied electromagnetic field determine the features of their subsequent dispersive interaction. Attempt to consider theoretically interrelation between parameters of the particles (the real part of their dielectric permeability and features of topology) and result of interaction with an applied electromagnetic wave falling on them has led to unexpected results.

Computational solution of the Maxwell's equations system for Relay's approximation (the length of a falling wave is much more the sizes of particles) has shown that electric field intensity on a surface of spherical form particles and a thin infinite cylinder is less than field intensity of a falling wave and decreases with growth of the real part of dielectric permeability. The exception is the cylinder with the asymmetrical form of section. In this case field intensity can increase in 28 times from a part of the cylinder with the minimum radius of curvature.

However, the main unexpectedness was showed by consideration of the toroidal form particles. It turns out that dramatic increasing of a falling field intensity (more than in $4 \times 10^{4}$ times) is reached at a certain ratio of the radiuses of tour and its generator with the real part of a dielectric constant. Such enormous increasing of a field power has all signs of a huge resonance and assumes drastic growth of the such particles interaction with each other and with external bodies even at insignificant level of their induction polarization. In other words, the external electromagnetic field can have induction-polarizing character.

\section{Experimental Results and Discussion}

Carbon nanoparticles fulleroid type received in the end of the past century name "astralenes", early using as effective for nanomodification of concretes, meet the requirements resulted above. Synthesis of the astralenes is sophisticate plasma-arc process with the subsequent technical-chemical excretion of tore-similar particles with the various sizes (mainly from 15 to 150 nanometers).

We have carried out the enrichment process of a great quantity of toriodal particles by special particles with shape ratio from 3:1 to $10: 1$. Considering that the value of dielectric permeability of fulleroid type nanocarbon particles is approximately 1,7-1.8 one would expect increasing of an external power field in thousands of times. It should be noted that practical operation with astralenes is extremely complicated in view of their ability to agglomeration (Figure 4).

The most effective way to separate of astralene clusters is intensive ultrasonic processing in the liquid environment at specific power of a signal more than $2-4 \mathrm{Wt} / \mathrm{cm}^{3}$. Introduction of astralenes into various composite materials thanks to powerful interaction effects at level of their mesostructure causes radical changes of the materials properties. Colloidal liquids at lower concentrated (less $10^{-6}$ mass \%) suspensions of astralenes in water or in organic liquids can be stable enough and demonstrate the strong non-linear optical properties, being thus a phototropic environment with the big running speed (less than $1 \mathrm{~ns}$ ). Presence of the astralenes into polymeric matrix of constructional and antifrictional carbon fiber reinforced plastics leads to consolidation of interphase binderfiller borders, thereby raises the operational properties of these composites. At the expense of consolidation of "molecular cavities» mechanical strength characteristics grow, and water absorption of polymeric matrixes decreases. Nonlinearity of electrophysical properties (conductivity) of the nanostructured polymer composites was revealed in response to powerful current impulses.

Cement concrete is effective example of astralelene modified material. At introduction astralens into concrete mixes a number of practically useful processes occurs:

1. The directional crystallization of a cement stone which is caused by features of cement hydration and pozzolanic additives near to strong field of heterogeneities as result of astralenes presence.

2. Increase concrete mixes fluidity at a stage of their preparation and the placement

3. Modification and consolidation of interphase borders: mineral binder-filler.

Initial phases of growth of lengthy structures in a water cement mix with astralenes is shown in Figure 5.

\section{References}

1. Figovsky O, Beilin D (2017) Green nanotechnology. Pan Stanford Publishing. USA, p. 539.

2. Figovsky O, Gumarov V (2018) Innovation systems: achievements \& problems. Lambert Academic Publishing. Mauritius, Germany, p. 646. 
3. Figovsky 0, Beilin D (2017) Advanced polymer concretes and composites. CRS Publishers, USA, p. 245.
4. Kudryavtsev, Figovsky O (2018) Nanomaterias based on soluble silicates. Lambert Academic Publishing, Mauritius, Germany, p. 241. (c) (i)

Creative Commons Attribution 4.0 International License

For possible submissions Click Here

\section{Submit Article}

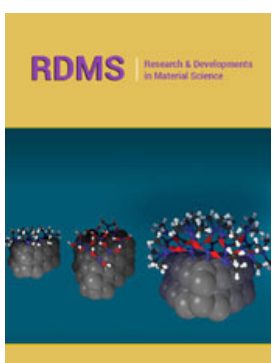

\section{Research \& Development in Material Science}

\section{Benefits of Publishing with us}

- High-level peer review and editorial services

- Freely accessible online immediately upon publication

- Authors retain the copyright to their work

- Licensing it under a Creative Commons license

- Visibility through different online platforms 